\title{
Langmuir
}

pubs.acs.org/Langmuir

(C) 2009 American Chemical Society

\section{Bioconjugation of Rod-Shaped Fluorescent Nanocrystals for Efficient Targeted Cell Labeling}

\author{
Alessandra Quarta, ${ }^{\ddagger, \|}$ Andrea Ragusa, ${ }^{\dagger, \|}$ Sasanka Deka, ${ }^{\star}$ Claudia Tortiglione,${ }^{\S}$ Angela Tino, \\ Roberto Cingolani, ${ }^{*}$ and Teresa Pellegrino ${ }^{*}, \dagger$ \\ ${ }^{\dagger}$ National Nanotechnology Laboratory of CNR-INFM and IIT Research Unit, via per Arnesano Km 5 , \\ 73100 Lecce, Italy, "Italian Institute of Technology, via Morego 30, 16163 Genoa, Italy, and ${ }^{\S}$ CNR, \\ Istituto di Cibernetica "E Caianiello", CNR, Via Campi Flegrei, 34, 80078 Pozzuoli, Italy. "These authors have \\ contributed equally to this paper.
}

Received May 22, 2009. Revised Manuscript Received July 13, 2009

\begin{abstract}
In the present work, we report a three-step approach for the functionalization of $\mathrm{CdSe} / \mathrm{CdS}$ core/shell and $\mathrm{CdSe} / \mathrm{CdS} /$ $\mathrm{ZnS}$ double-shell quantum rods (QRs) with either biotin or folic acid. We carried out an in vitro study on cultured cells and fixed tissue sections in which the biofunctionalized QRs were compared with the more traditional $\mathrm{CdSe} / \mathrm{ZnS}$ quantum dots (QDs), which were also functionalized with either biotin or folic acid. The QR and the QD samples exhibited the same specificity toward the targeting cells. In addition, due to the enhanced photoluminescence of the QRs with respect to QDs, a lower amount of rods was required to image cells. In immuno-localization experiments on rat brain tissue sections, biotin-functionalized QRs have shown the typical protein localization patterns expected both for neuronal enolase NSE and actin, confirming the specificity of the interaction of QRs with avidin, and the feasibility of these materials as fluorescent probes in tissue staining. In this specific targeting study, we could assess via the MTT test, a cell viability assay, the lower toxicity of the $\mathrm{CdSe} / \mathrm{CdS} / \mathrm{ZnS}$ QRs with respect to $\mathrm{CdSe} / \mathrm{CdS} \mathrm{QRs}$.
\end{abstract}

\section{Introduction}

Advances in semiconductor colloidal nanocrystals, ranging from spherical nanocrystals, also called quantum dots (QDs), to rod-like nanocrystals, also known as quantum rods (QRs), provide a new set of materials with unique and tunable optical properties. ${ }^{1}$ In the latest years, QDs, suitably coated for biological applications, have become commercially available. Since then, this novel class of fluorophores has been widely explored both in vitro and in vivo, and their peculiar properties have substantially overcome the limitations of traditional organic fluorophores. ${ }^{2-5}$

The photostability and the advantageous signal-to-noise ratio achievable with QDs make them ideal probes for single molecule tracking studies. ${ }^{6}$ Furthermore, the excellent photostability of QDs can be particularly useful for continuous irradiation and 3D optical acquisition under a confocal microscopy, where image reconstruction and quality are strongly limited by photodegradation of organic fluorophores. 7,8

The high brightness and sensitivity offered by QDs combined with the availability of a large number of well-separated colors, all excitable with one single source, make them suitable for multiplexed cellular imaging. ${ }^{9,10}$ Recently, much effort has been

*Corresponding author. E-mail: teresa.pellegrino@unile.it.

(1) Alivisatos, A. P. Science 1996, 271(5251), 933-937.

(2) Bruchez, M.; Moronne, M.; Gin, P.; Weiss, S.; Alivisatos, A. P. Science 1998, 281(5385), 2013-2016.

(3) Chan, W. C. W.; Maxwell, D. J.; Gao, X. H.; Bailey, R. E.; Han, M. Y.; Nie,

S. M. Curr. Opin. Biotechnol. 2002, 13(1), 40-46.

(4) Chan, W. C. W.; Nie, S. M. Science 1998, 281(5385), 2016-2018.

(5) Wang, F.; Tan, W. B.; Zhang, Y.; Fan, X. P.; Wang, M. Q. Nanotechnology 2006, 17(1), R1-R13.

(6) Courty, S.; Luccardini, C.; Bellaiche, Y.; Cappello, G.; Dahan, M. Nano Lett. 2006, 6(7), 1491-1495.

(7) Tokumasu, F.; Dvorak, J. J. Microscopy (Oxford) 2003, 211, 256-261.

(8) Lacoste, T. D.; Michalet, X.; Pinaud, F.; Chemla, D. S.; Alivisatos, A. P.; Weiss, S. Proc. Natl. Acad. Sci. U.S.A. 2000, 97(17), 9461-9466.

(9) Smith, A. M.; Dave, S.; Nie, S. M.; True, L.; Gao, X. H. Expert Rev. Mol. Diagn. 2006, 6(2), 231-244.

(10) Medintz, I. L.; Uyeda, H. T.; Goldman, E. R.; Mattoussi, H. Nat. Mater. 2005, 4(6), 435-446. directed toward the development of biocompatible nanocrystals endowed with signaling molecules which could act as targeting cellular probes. ${ }^{10-13}$ So far, numerous cellular compartments and proteins have been labeled with functionalized QDs, both in living and fixed cells, and such a list includes nuclei, mitochondria, microtubules, actin filaments, cytokeratin, endocytic compartments, mortalin, and chaperonin proteins. ${ }^{14-19}$ The idea to employ QDs as cell markers was based not only on their superior optical properties but also on the discovery that they can be internalized by cells, either via receptor-mediated ${ }^{4}$ or by nonspecific endocytosis. ${ }^{17,20-22}$

Receptor-mediated endocytosis is a process promoted by a specific key/keyhole type receptor-ligand binding interaction. To this aim, it is important that the ligand responsible for the recognition of the receptor is still able to find and bind its target

(11) Michalet, X.; Pinaud, F. F.; Bentolila, L. A.; Tsay, J. M.; Doose, S.; Li, J. J.; Sundaresan, G.; Wu, A. M.; Gambhir, S. S.; Weiss, S. Science 2005, 307(5709), $538-544$.

(12) Alivisatos, A. P.; Gu, W. W.; Larabell, C. Ann. Rev. Biomed. Eng. 2005, 7 $55-76$.

(13) Pinaud, F.; Michalet, X.; Bentolila, L. A.; Tsay, J. M.; Doose, S.; Li, J. J.; Iyer, G.; Weiss, S. Biomaterials 2006, 27(9), 1679-1687.

(14) Chen, F. Q.; Gerion, D. Nano Lett. 2004, 4(10), 1827-1832.

(15) Hoshino, A.; Fujioka, K.; Oku, T.; Nakamura, S.; Suga, M.; Yamaguchi, Y.; Suzuki, K.; Yasuhara, M.; Yamamoto, K. Microbiol. Immunol. 2004, 48(12), 985-994.

(16) Mansson, A.; Sundberg, M.; Balaz, M.; Bunk, R.; Nicholls, I. A.; Omling, P.; Tagerud, S.; Montelius, L. Biochem. Biophys. Res. Commun. 2004, 314(2), 529534 .

(17) Hanaki, K.; Momo, A.; Oku, T.; Komoto, A.; Maenosono, S.; Yamaguchi, Y.; Yamamoto, K. Biochem. Biophys. Res. Commun. 2003, 302(3), 496-501.

(18) Kaul, Z.; Yaguchi, T.; Kaul, S. C.; Hirano, T.; Wadhwa, R.; Taira, K. Cell Res. 2003, 13(6), 503-507.

(19) Ishii, D.; Kinbara, K.; Ishida, Y.; Ishii, N.; Okochi, M.; Yohda, M.; Aida, T. Nature 2003, 423(6940), 628-632.

(20) Jaiswal, J. K.; Mattoussi, H.; Mauro, J. M.; Simon, S. M. Nat. Biotechnol. 2003, 2l(1), 47-51.

(21) Parak, W. J.; Boudreau, R.; Le Gros, M.; Gerion, D.; Zanchet, D.; Micheel, C. M.; Williams, S. C.; Alivisatos, A. P.; Larabell, C. Adv. Mater. 2002, 14(12), $882-885$.

(22) Michalet, X.; Pinaud, F.; Lacoste, T. D.; Dahan, M.; Bruchez, M. P.; Alivisatos, A. P.; Weiss, S. Single Mol. 2001, 2(4), 261-276. 
even after conjugation to the nanocrystal. Indeed, in the case of a QD-ligand complex, it has been shown that the QD attached to the ligand does not alter the recognition ability of the ligand to the receptor because of the steric hindrance effect. ${ }^{20,23}$

Another issue related to the employment of inorganic nanocrystals (NCs) in biological studies is their toxicity. Traditional II/VI NCs contain elements such as $\mathrm{Cd}$, Se, and $\mathrm{Zn}$, which are bound by metallothionein proteins and may be excreted slowly or sequestered in vivo in adipose and other tissues. ${ }^{24}$ In general, there are discrepancies in the current literature regarding the toxicity of QDs. These can be attributed to different factors, such as the variety of QD dosage/exposure concentrations, the individual features of QDs (size, charge, coating structure, and mechanical stability), ${ }^{25}$ and the environmental conditions (oxidative or photolytic surroundings). ${ }^{26}$

To reduce QD toxicity, nanocrystal technology is moving toward two distinct directions. One direction is the development of Cd-free fluorescent nanocrystals. ${ }^{27,28}$ As an example, Pradhan et al. synthesized $\mathrm{Cu}$ or $\mathrm{Mn}$ doped $\mathrm{ZnSe}$ quantum dots, emitting from the blue to the red region of the visible spectrum and with appreciable quantum yields. ${ }^{27}$ However, the Cd-free NCs obtained so far do not possess optical properties comparable to those of more classical type II/VI NCs. Henceforth, while waiting for new generations of Cd-free QDs, a second direction to reduce toxicity is by improving the optical properties of the type II/VI $\mathrm{NCs}^{29}$ in order to get much brighter nanoparticles and thus lowering the amount of NCs needed for bioprobing.

Within this frame, rod-shaped nanocrystals represent a new class of fluorescent particles with stronger luminescence and larger absorption cross-section compared to those of QDs. ${ }^{30}$ Furthermore, quantum rods (QRs) exhibit linearly polarized emission, unlike the plane polarized emission from a single QD, and large surface areas, which should facilitate and improve the functionalization with biomolecules. ${ }^{31}$ All of these features make QRs appealing fluorescent tools for biological and medical applications.

So far, only few reports have described the use of QRs as in vitro imaging probes. ${ }^{32-34}$ The group of Prasad and that of Alivisatos independently showed that $\mathrm{CdSe} / \mathrm{CdS} / \mathrm{ZnS}$, prepared by growing a $\mathrm{CdS} / \mathrm{ZnS}$ graded shell over CdSe dots, conjugated to transferrin, folic acid, or antibodies could actively target tumor cells, allowing for fluorescent labeling and even two-photon imaging. ${ }^{34,35}$ Despite their superior optical properties, which may also allow improvements for deep tissue imaging, to the best

(23) Delehanty, J. B.; Mattoussi, H.; Medintz, I. L. Anal. Bioanal. Chem. 2009, 393(4), 1091-1105.

(24) Colvin, V. L. Nat. Biotechnol. 2003, 21(10), 1166-1170.

(25) Lewinski, N.; Colvin, V.; Drezek, R. Small 2008, 4(1), 26-49.

(26) Hardman, R. Environ. Health Perspect. 2006, 114(2), 165-172.

(27) Pradhan, N.; Goorskey, D.; Thessing, J.; Peng, X. G. J. Am. Chem. Soc. 2005, 127(50), 17586-17587.

(28) Liu, W.; Howarth, M.; Greytak, A. B.; Zheng, Y.; Nocera, D. G.; Ting,

A. Y.; Bawendi, M. G. J. Am. Chem. Soc. 2008, 130(4), 1274-1284.

(29) Reiss, P.; Protiere, M.; Li, L. Small 2009, 5(2), 154-168.

(30) Htoon, H.; Hollingsworth, J. A.; Dickerson, R.; Klimov, V. I. Phys. Rev. Lett. 2003, 91(22), 227401.

(31) Carbone, L.; Nobile, C.; De Giorgi, M.; Della Sala, F.; Morello, G.; Pompa, P.; Hytch, M.; Snoeck, E.; Fiore, A.; Franchini, I. R.; Nadasan, M.; Silvestre, A. F.; Chiodo, L.; Kudera, S.; Cingolani, R.; Krahne, R.; Manna, L. Nano Lett. 2007, 7(10), 2942-2950.

(32) Yong, K. T.; Qian, J.; Roy, I.; Lee, H. H.; Bergey, E. J.; Tramposch, K. M.; He, S. L.; Swihart, M. T.; Maitra, A.; Prasad, P. N. Nano Lett. 2007, 7(3), 761-765.

(33) Fu, A. H.; Gu, W. W.; Boussert, B.; Koski, K.; Gerion, D.; Manna, L.; Le Gros, M.; Larabell, C. A.; Alivisatos, A. P. Nano Lett. 2007, 7(1), 179-182.

(34) Yong, K. T.; Roy, I.; Pudavar, H. E.; Bergey, E. J.; Tramposch, K. M.; Swihart, M. T.; Prasad, P. N. Adv. Mater. 2008, 20(8), 1-6.

(35) Deka, S.; Quarta, A.; Falqui, A.; Lupo, M. G.; Lanzani, G.; Giannini, C.; Cingolani, R.; Pellegrino, T.; Manna, L. J. Am. Chem. Soc. 2008, 131(8), 29482958 . of our knowledge the efficient use of QRs as contrast agents for tissue immunostaining has not been reported yet. Standard protocols relying on colorimetric detection systems or fluorescence-based secondary antibodies present several disadvantages, such as nonspecific background and low photostability/fading of the organic fluorophores (Cy5, Alexafluor, and so on) coupled to the antibody. Thus, the availability of bioconjugated QRs to be used as probes for tissue imaging with more specific and higher resolution detection could greatly improve bioimaging.

In the attempt to prepare new biocompatible nanoparticles with enhanced optical properties, reduced toxicity, and suitable surface functionalization for cell targeting, we have carried out an in vitro comparative study of biotin and folic acid functionalized $\mathrm{CdSe} / \mathrm{CdS}$ and $\mathrm{CdSe} / \mathrm{CdS} / \mathrm{ZnS}$ QRs with respect to similarly functionalized $\mathrm{CdSe} / \mathrm{ZnS}$ QDs. The rods employed in this study were synthesized by a new seeded-type growth approach recently reported by our group. ${ }^{35}$ The feasibility to employ the resulting functionalized nanocrystals, which have higher quantum yields compared to traditional $\mathrm{CdSe} / \mathrm{ZnS}$ QDs, was exploited by targeting cultured cells and fixed tissue sections. The results reported in the present work can be summarized as follows:

(1) Biotin-conjugated nanocrystals were targeted to HeLa cells, exploiting the biotin-avidin interaction, and the specific intracellular NC cellular uptake was monitored by fluorescence microscopy under different conditions. Moreover, a similar approach was used to test immunolocalization protocols on rat brain slides, confirming superior optical features.

(2) Folate-conjugated nanocrystals (both $\mathrm{CdSe} / \mathrm{CdS}$ and $\mathrm{CdSe} / \mathrm{CdS} / \mathrm{ZnS} \mathrm{QRs})$ were targeted to folatereceptor positive cell lines, such as human epidermal carcinoma KB cells, and the specific internalization by receptor-mediated endocytosis was compared with that of bare QRs.

(3) Finally, the cytotoxic effect of the three bioconjugated nanoparticles (QDs and both types of QRs) on tumor cells was also investigated.

(4) Henceforth, for simplicity the nanocrystals will be referred to with the following abbreviations: QDs instead of core/shell CdSe/ZnS QDs, CS rods instead of core/shell $\mathrm{CdSe} / \mathrm{CdS}$ rods, and CSS rods instead of core/shell/shell $\mathrm{CdSe} / \mathrm{CdS} / \mathrm{ZnS}$ rods.

\section{Results}

Preparation and Characterization of Biotin- and FolateConjugated Nanocrystals. The synthesis and the use of CS and CSS rods as nonspecific fluorescent cellular probes has been recently reported by Deka et al. as an alternative to more traditional QDs. ${ }^{35}$ Those rods were synthesized by means of a new seeded-type growth approach which produces particles with narrow distribution of lengths and diameters, yielding rods with high quantum yield both in organic and aqueous solvents. Also, while keeping high colloidal stability, the larger surface area of one-dimentional QRs compared to zero-dimentional QDs should allow the covalent binding of a higher number of biomolecules per nanocrystal, such as proteins and antibodies, which make them ideal probes for specific labeling, both in vitro and in vivo.

The biotin and folic acid functionalization of the NCs used in this study is schematically drawn in Figure 1. The as-synthesized QDs have a diameter of $6 \mathrm{~nm}$, while CS and CSS QRs have a diameter of $5 \mathrm{~nm}$ and a length of $22 \mathrm{~nm}$, as determined by transmission electron microscopy (TEM) analysis. The samples 


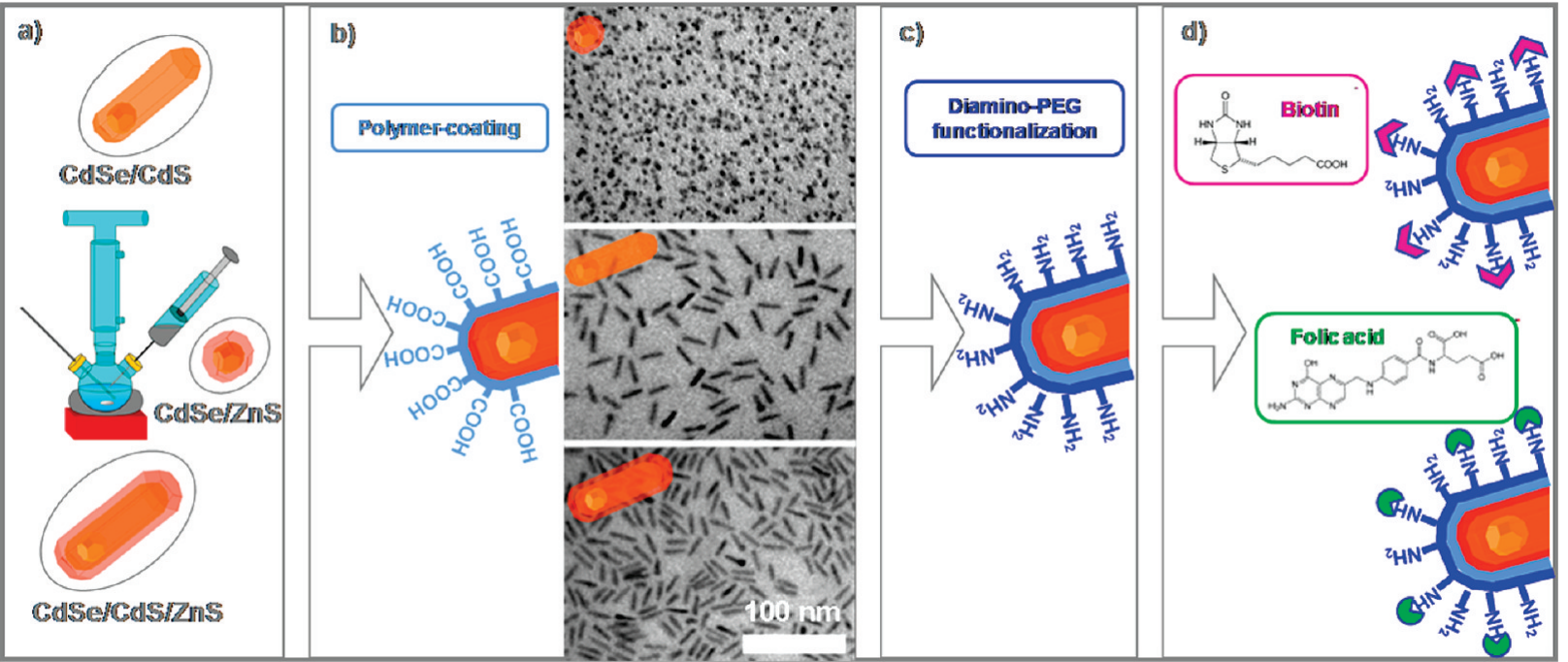

Figure 1. Synthetic route for the preparation of the biotinylated and the FA(folic acid)-conjugated nanocrystals. (a) The as-synthesized colloidal nanocrystals were (b) transferred into water through a polymer-coating procedure; ${ }^{36,37}$ (c) diamino-PEG molecules were attached to the polymeric shell to improve biocompatibility and to act as spacers, ${ }^{38,39}$ (d) biotin and folic acid were covalently bound to the outstretched amino groups of PEG.

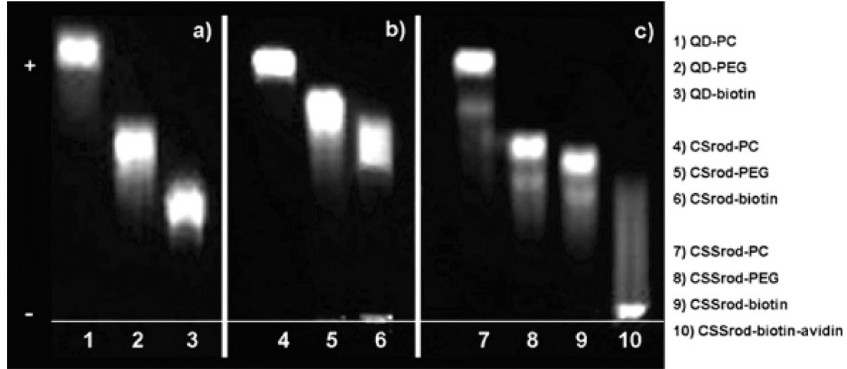

Figure 2. Gel electrophoretic characterization of both spherical and rod-shaped nanocrystals functionalized with biotin. Panel a reports the migration of QDs, while panels $b$ and $c$ present the conjugation products for CS- and CSS-QRs, respectively. In each panel, the first lane refers to the polymer-coated samples, the second lane to the diamino-PEG functionalized nanoparticles, and the third lane to the biotin conjugates. Line 10 in panel $\mathrm{c}$ corresponds to the avidin-biotin-CSS rods complex.

were made water-soluble by means of a polymer-coating procedure and purified by ultracentrifugation in a sucrose gradient, ${ }^{36,37}$ resulting in monodispersed and highly stable nanocrystals, as shown in the TEM images of the polymer-coated particles (Figure 1). Diamino-poly(ethylene glycol) (PEG) molecules were then attached through EDC ( $N$-(3-dimethylaminopropyl)- $N^{\prime}$ ethyl-carbodiimide) chemistry to the carboxyl group of the polymer to reduce nonspecific cellular uptake ${ }^{38}$ and to act as linkers for the subsequent conjugation of biotin or folic acid through the amino terminated moiety of the PEG molecule ${ }^{39}$ (see the Experimental Section for details).

Figure 2 reports the gel electrophoretic migration patterns of the polymer-coated samples (lines 1, 4, and 7), the diamino-PEG functionalized nanoparticles (lines 2, 5, and 8), and the biotin-

(36) Pellegrino, T.; Manna, L.; Kudera, S.; Liedl, T.; Koktysh, D.; Rogach, A L.; Keller, S.; Radler, J.; Natile, G.; Parak, W. J. Nano Lett. 2004, 4(4), 703-707.

(37) Di Corato, R.; Quarta, A.; Piacenza, P.; Ragusa, A.; Figuerola, A.; Buonsanti, R.; Cingolani, R.; Manna, L.; Pellegrino, T. J. Mater. Chem. 2008, 18(17), 1991-1996.

(38) Chang, E.; Thekkek, N.; Yu, W. W.; Colvin, V. L.; Drezek, R. Small 2006, 2(12), 1412-1417.

(39) Sperling, R. A.; Pellegrino, T.; Li, J. K.; Chang, W. H.; Parak, W. J. Adv Funct. Mater. 2006, 16(7), 943-948. conjugated nanocrystals (lines 3, 6, and 9) for all three types of nanoparticles (QDs and both QRs). For each type of nanocrystal, the polymer-coated sample was always the less retained, running toward the positive pole because of the presence of the negatively charged surface due to the carboxylic groups. The addition of PEG molecules reduced the negative charge of the nanoparticle and increased the nanocrystals size, retarding the nanoparticle gel mobility. A similar electrophoretic behavior was observed for the biotin conjugates. To confirm the successful biotin attachment to the nanoparticles and the preservation of its binding ability, avidin was also added to the biotin-CSS conjugates to induce the formation of large complexes. Since avidin has four binding pockets, probably more than one biotin-nanoconjugate could bind to the same avidin molecule, leading to the formation of structures with sizes too big to run through the gel (Figure 2, line 10). The same behavior was also observed for the other conjugates (data not shown).

The size increase was also determined in solution by dynamic light scattering (DLS) (Table S1, Supporting Information). The average hydrodynamic diameter of the nanoparticles in borate saline buffer ( $\mathrm{pH}$ 9) at each step of functionalization was measured, showing a progressive increase in size and confirming the electrophoretic behavior. Zeta-potential measurements also showed a consistent charge increase after PEG functionalization, while the charge did not vary significantly after biotin conjugation (Table S1, Supporting Information).

Water-solubilization and bioconjugation did not affect the optical properties of the NCs, and no significant changes in their $\mathrm{UV}-$ vis and photoluminescence (PL) spectra were detected (Figure S1, Supporting Information). All of the samples have a PL maximum emission peak at $615 \mathrm{~nm}$. Orange-emitting QDs and QRs were chosen in order to increase the contrast between the greenish cell autofluorescence and the probe. As expected, the PL quantum yield $(\mathrm{QY})$ of the water-soluble nanoparticles was lower than that of the corresponding samples dissolved in organic solvent. Nevertheless, the PL quantum efficiency of both QRs was still much higher than that of the QDs $(\sim 10 \%)$, while that of the CSS QRs $(\sim 43 \%)$ was only slightly higher than that of the CS QRs $(\sim 39 \%)$.

Folic acid conjugation on the amino PEG derivatives was also carried out through EDC chemistry. However, trials at different 
concentrations had to be done before reaching a good compromise, which yielded stable and fluorescent nanocrystals since attachment of the ligand often resulted in quenching and precipitation of the conjugates. To avoid these problems, we found that very dilute solutions had to be used $(0.1$ and $0.01 \mu \mathrm{M}$ for QRs and activated FA, respectively). Still, the amount of FA conjugated under these conditions was sufficient to induce specific cell recognition as shown later. TEM imaging of the QRs showed that, after conjugation with a suitable amount of folic acid, the nanorods remained monodispersed and maintained their original optical properties without showing any significant variation with respect to the corresponding diamino-PEG precursors (Figure S2, Supporting Information).

Successful conjugation of the ligand was confirmed by gel electrophoresis, from which it was noticed that folic acid attachment retarded the front edge of the migration band of the samples slightly (Figure 3), probably due to the low amount of ligand that could be conjugated in the reaction conditions followed to avoid the quenching problem mentioned above.

DLS measurements also confirmed the observations by gel electrophoresis, as the hydrodynamic radius of both QR-FA

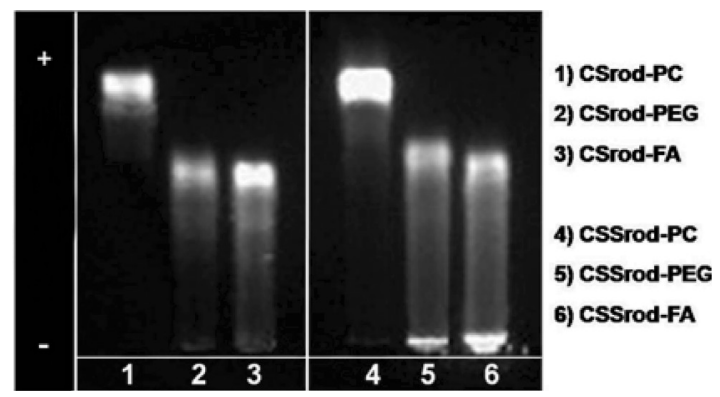

Figure 3. Gel electrophoresis characterization of the folate-rod conjugate. On the same gel, the polymer coated (lane 1), the diamino-PEG functionalized (lane 2), and the folate-conjugated (lane 3) samples were run in parallel.

Table 1. DLS and Zeta-Potential Measurements in Borate Buffer (pH 9) of the CS- and CSS-QRs after Polymer Coating, PEG Linking, and FA Conjugation

\begin{tabular}{llllll}
\hline \multicolumn{3}{c}{ DLS } & & \multicolumn{2}{c}{ zeta-potenlial } \\
\cline { 1 - 2 } \cline { 5 - 6 } \cline { 5 - 6 } & CSrod $(\mathrm{nm})$ & CSSrod $(\mathrm{nm})$ & & CSrod $(\mathrm{mV})$ & CSSrod $(\mathrm{mV})$ \\
\hline PC & $24.3 \pm 1.7$ & $30.5 \pm 1.2$ & & $-25.0 \pm 1.0$ & $-21.4 \pm 3.1$ \\
PEG & $34.8 \pm 1.7$ & $39.1 \pm 0.5$ & & $-11.2 \pm 0.4$ & $-9.5 \pm 0.2$ \\
FA & $36.1 \pm 1.2$ & $41.3 \pm 1.3$ & & $-15.0 \pm 1.6$ & $-13.6 \pm 1.3$
\end{tabular}

samples showed a modest increase $(\sim 2 \mathrm{~nm})$ with respect to the corresponding QR-PEG conjugates (Table 1). Zeta potential measurements gave a more clear response about the successful conjugation. In fact, both samples showed an increased negative charge after FA conjugation, which is likely due to the decreased number of primary PEG amines on the nanocrystal surface (used for FA conjugation) and to the additional free carboxylic groups of the appended folate ligands (Table 1).

Cell Studies Based on Biotin-Conjugated Nanocrystals. In order to specifically label the cell surface with biotin-conjugated nanocrystals, a three-step procedure was introduced: first, NHSbiotin (biotin 3-sulfo- $N$-hydroxysuccinimide ester) molecules were anchored to the HeLa cell membrane proteins. Then, a solution of avidin was added to the medium to form biotinavidin complexes at the cell surface. Both steps were performed at $4{ }^{\circ} \mathrm{C}$ to inhibit endocytosis. Finally, nanocrystals (both QDs and QRs) were supplied to cultured cells under two different conditions and incubation times, for $1 \mathrm{~h}$ at $4{ }^{\circ} \mathrm{C}$ and for $24 \mathrm{~h}$ at $37^{\circ} \mathrm{C}$.

It is worth noticing that, since the three types of nanocrystals used in the present work differ from each other in terms of size, shape, and composition, we have administered NC samples always at the same $\mathrm{Cd}$ concentration (measured by elemental analysis by using an AES-ICP spectrometer), which corresponds to an absolute number of QRs always lower than that used for QDs. Doping HeLa cells with NCs for $1 \mathrm{~h}$ at $4{ }^{\circ} \mathrm{C}$ prevented endocytosis of the $\mathrm{NC}$-biotin-avidin-biotin complex, as shown by the fluorescence accumulated at the cell surface (Figure 4). In Figure $4 \mathrm{a}$, which shows the cells treated with $\mathrm{CS}-\mathrm{QR}-$ biotin conjugates, the fluorescence appears in restricted regions of the membrane (the confocal pictures were acquired at a height showing the cell membranes in focus), probably due to the localization of the surface proteins to which the NHS-biotin was initially bound. The same result was obtained with CSS QRs (data not shown). As a control experiment, diamino-PEG functionalized rods were administered to the cells under the same experimental conditions, but no cell-bound fluorescence was detected (Figure 4b).

By contrast, when the NC incubation was carried out for $24 \mathrm{~h}$ at $37^{\circ} \mathrm{C}$, the biotin-avidin-biotin-nanocrystal complexes were clearly internalized by the cells (Figure 5). Although the fluorescence signal was widely spread inside the cell, a stronger intensity could be detected around the cell nucleus, likely due to intravesicle storage of the nanocrystals. From a qualitative analysis, the intensity of the fluorescence signal looks higher in the specimens doped with QRs rather than with QDs (the confocal images were acquired by using in all cases the same confocal setup; see Experimental Section for details). A quantitative assay was

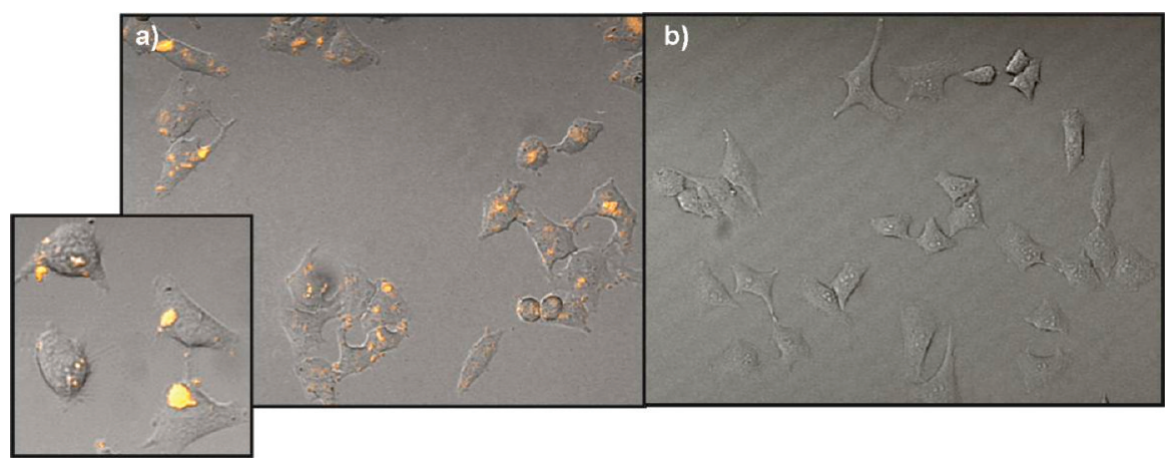

Figure 4. Confocal microscopy images of living HeLa cells marked with CS-QRs after $1 \mathrm{~h}$ of incubation at $4{ }^{\circ} \mathrm{C}$. HeLa cells were first treated with NHS-biotin, then avidin, and finally labeled with (a) CS-biotin QRs and (b) CS-PEG QRs at $50 \mu \mathrm{M}$ Cd concentration. In the inset of panel a, a higher magnification of the same field is reported. 


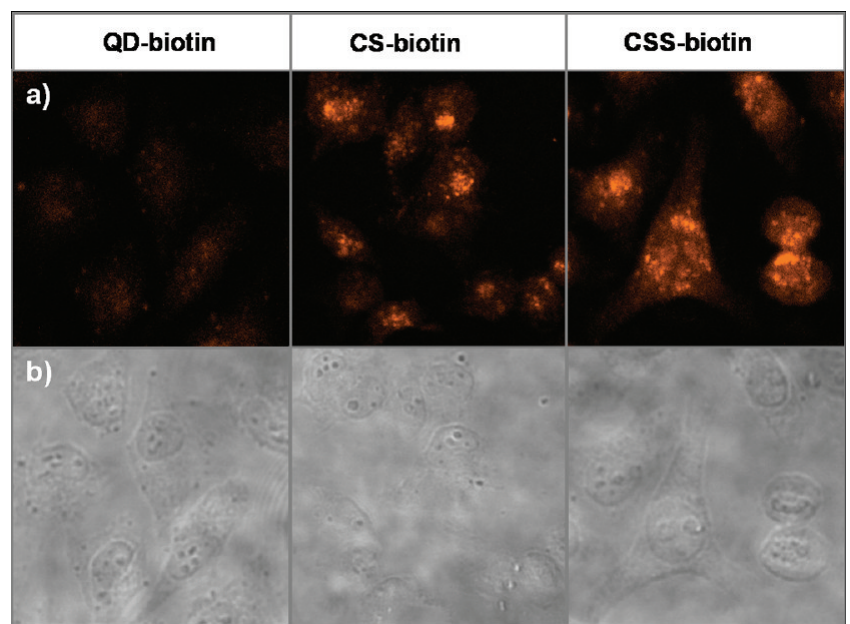

Figure 5. Confocal microscopy images of fixed HeLa cells, treated with NHS-biotin and avidin and then labeled with QD- and QRbiotin conjugates at a $\mathrm{Cd}$ concentration equal to $50 \mu \mathrm{M}$ for $24 \mathrm{~h}$ at $37^{\circ} \mathrm{C}$. Images were acquired (a) under excitation by the $488 \mathrm{~nm}$ line of an argon laser and (b) under bright field exposure.

performed to determine whether the increased fluorescence was due to a higher number of internalized QRs or to their increased luminescence compared to that of the QDs. Cells doped for $24 \mathrm{~h}$ with the biotinylated nanocrystals (both QDs and QRs) were washed, detached from the substrate by trypsin treatment, and counted in a cell-counting chamber. The PL spectrum of each sample was then recorded and normalized to the number of cells.

It is significant to emphasize that by administering to the cell a given amount of cadmium, if in the form of QRs, this corresponded to a lower number of QR nanoparticles compared to that of QDs. We actually found the highest PL intensities in the cell samples that had internalized the CSS-biotin conjugates and the lowest PL intensities in cell samples that had internalized the corresponding QDs (Figure 6a). To correlate PL intensities to the amount of nanocrystals taken up by the cells, we carried out elemental analysis (based on the intracellular Cd concentration) on these cell suspensions. The values we found, expressed as $\mathrm{Cd}$ concentration, were converted into nanoparticle concentration and normalized to the initial number of cells (Figure 6b) (see Experimental Section for details). In the case of QRs, despite the fact that they displayed the highest intracellular Cd concentration, the corresponding nanoparticle concentration was almost half of that found for cells that had internalized QDs because of the much bigger size of QRs with respect to that of QDs. Nonetheless, the PL signal from the cells that had internalized the QRs was much higher than that of cells which had internalized the QDs.

The ratio between the intracellular NP(nanoparticle) concentration and the initial NP concentration administered to the cells provided a clear indication of the higher internalization efficiency of the QRs compared to that of QDs (Figure 6b, column 6). As already observed by other groups, the shape of the nanoparticles plays a key role in the cell internalization process. ${ }^{40,41}$ As an example, in a comparative study of the uptake behavior of gold nanorods and spherical gold nanoparticles (the latter $50 \mathrm{~nm}$ in diameter), Chan and co-workers had found a reduced uptake of gold rods with respect to the spherical nanoparticles, in contrast

(40) Decuzzi, P.; Pasqualini, R.; Arap, W.; Ferrari, M. Pharm. Res. 2009, 26(1), 235-243.

(41) Chithrani, B. D.; Ghazani, A. A.; Chan, W. C. W. Nano Lett. 2006, 6(4), $662-668$. a)
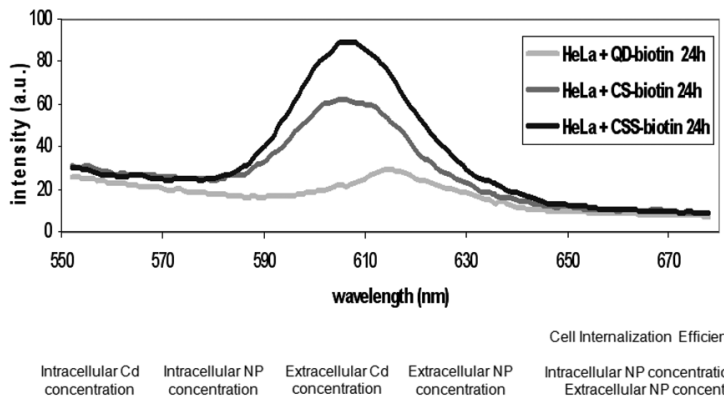

Intracellular NP
concentration

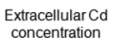

Extracellular NP
concentration

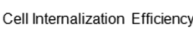

Sample

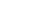

$$
\begin{aligned}
& (\mathrm{nM}) \\
& 1.45 \\
& 0.84
\end{aligned}
$$$$
\begin{gathered}
(\mu \mathrm{M}) \\
50 \\
50
\end{gathered}
$$

CSrod-biotin

9.2

9.3

$(\mathrm{nM})$
39.9
4.54
4.54

Intracellular NP concentration ! Extracellular NP concentration

$\%$

18.6

18.4

Figure 6. (a) PL emission curves of the nanocrystal-biotin conjugates (both QDs $\left(\lambda_{\max }\right.$ of emission $\left.615 \mathrm{~nm}\right)$ and QRs $\left(\lambda_{\max }\right.$ of emission $609 \mathrm{~nm}$ )) internalized by the cells after $24 \mathrm{~h}$ of incubation. The PL intensities were normalized to the number of cells (each PL curve corresponds to the same number of cells doped with the different biotin functionalized nanoparticle samples). (b) Table reports the corresponding intracellular $\mathrm{Cd}$ concentration (second column); the Cd concentration was then converted in nanoparticle concentration (third column); the initial $\mathrm{Cd}$ administered to the cells (third column); and the corresponding initial nanoparticle concentration (fourth column). The cell internalization efficiency (sixth column) is given as the ratio between the intracellular nanoparticle concentration and the initial extracellular nanoparticle concentration administered to the cells.

with our study in which QRs were internalized more efficiently than QDs. ${ }^{41}$ Such different findings might be attributed to the different types of nanoparticles studied in the two cases, which were characterized for instance by different surface functionalization (in our study, we covalently linked the biotin to the nanoparticle surface, while Chan and co-workers had mainly absorbed the transferrin to the surface of the gold nanorods) and additionally to the different cell lineages on which the particles were tested.

Our results indicate clearly that suitably functionalized fluorescent nanorods can be delivered to cells more efficiently than QDs and most remarkably that a lower amount of rods than QDs is required to image cells because of the improved optical properties of QRs with respect to those of traditional QDs (Figure 6b).

To investigate the toxicity of the biotin functionalized QRs, a cytotoxicity assay (standard MTT test) was carried out on the QD- and the QR-biotin conjugates supplied to HeLa cells for $24 \mathrm{~h}$ at two different $\mathrm{Cd}$ concentrations ( 5 and $50 \mu \mathrm{M})$ (Figure 7). As expected, higher $\mathrm{Cd}$ concentrations corresponded to an increased toxicity of the conjugates. At both values of $\mathrm{Cd}$ concentration, the CSS rods showed the lowest cytotoxicity, while the highest corresponded to the CS rods. These data may be explained by taking into consideration the structure and the composition of the nanocrystals used in this study. In CSS rods, the ZnS shell grown around the $\mathrm{CdSe} / \mathrm{CdS}$ rods screens the nanocrystal surface, thus reducing the release of $\mathrm{Cd}$ compared to that in bare $\mathrm{CdSe} /$ $\mathrm{CdS}$ rods. A lower amount of free $\mathrm{Cd}$ in solution or inside the cells certainly reduces cell mortality. ${ }^{25,42,43}$

In the case of QDs, in which a $\mathrm{ZnS}$ shell surrounds the spherical CdSe core, as the CSS rods, the MTT assay showed a higher toxicity compared to CSS rods. This data can likely suggests that if we administer to the cells the same $\mathrm{Cd}$ amount contained in nanoparticles of different shape and size, a greater number of the smallest particles will be available in the cell medium,

(42) Derfus, A. M.; Chan, W. C. W.; Bhatia, S. N. Nano Lett. 2004, 4(1), 11-18.

(43) Kirchner, C.; Liedl, T.; Kudera, S.; Pellegrino, T.; Javier, A. M.; Gaub, H. E.; Stolzle, S.; Fertig, N.; Parak, W. J. Nano Lett. 2005, 5(2), 331-338. 


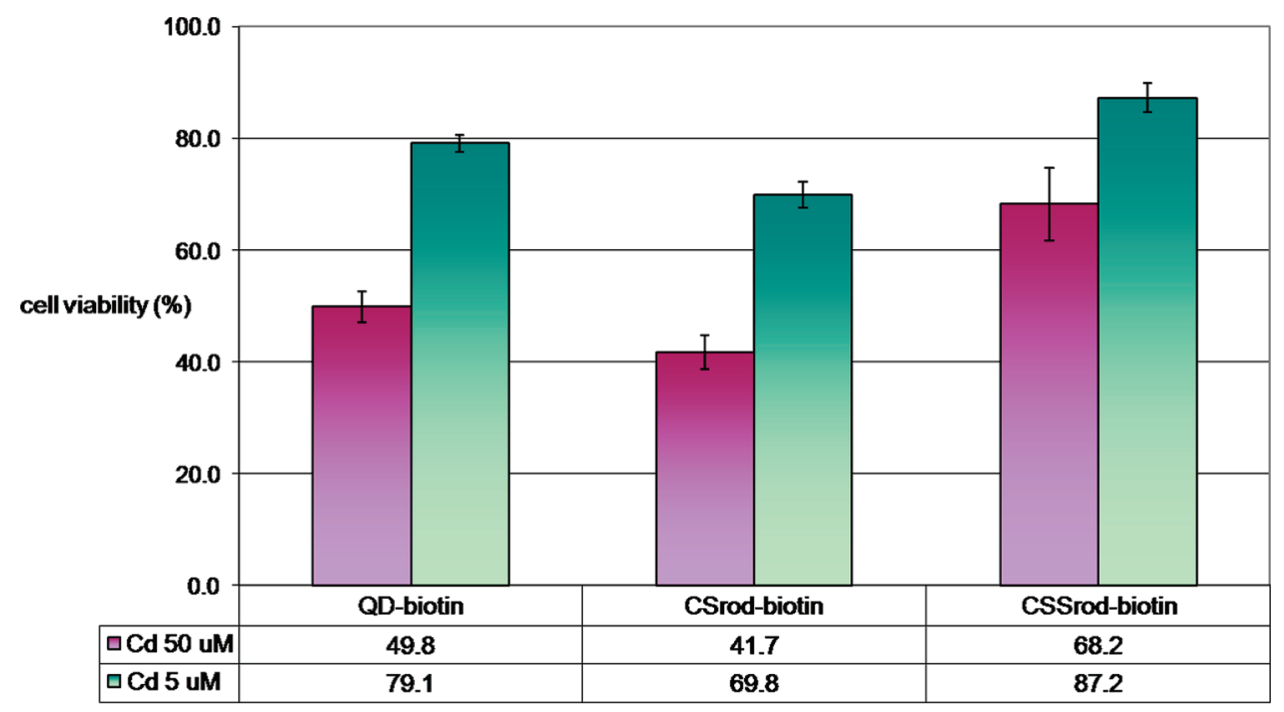

Figure 7. Cytotoxicity assay performed on HeLa cells incubated for $24 \mathrm{~h}$ with QD- and QR-biotin conjugates at two different concentrations (5 and $50 \mu \mathrm{M} \mathrm{Cd})$.
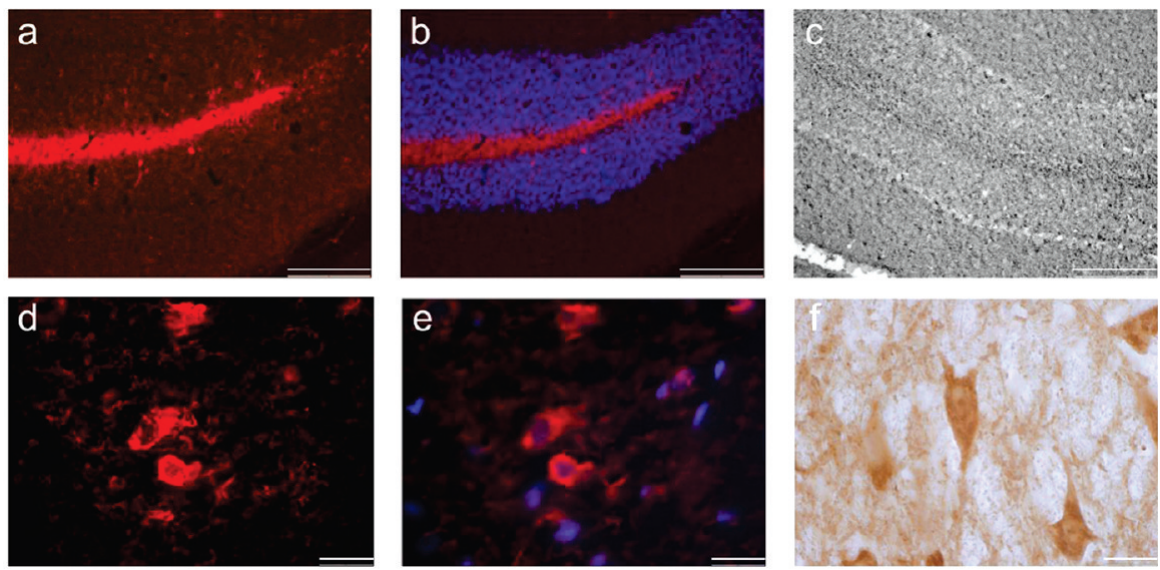

Figure 8. Fluorescence optical microscopy images from rat brain cryosections $14 \mu \mathrm{m}$ thick. (a-c) Rat cerebellum NSE immunoreactivity and $(\mathrm{d}-\mathrm{f})$ rat brain actin immunoreactivity. The immunoreaction was revealed with $(\mathrm{a}, \mathrm{b}, \mathrm{d}, \mathrm{e}) \mathrm{CS}-$ biotin and $(\mathrm{c}, \mathrm{f}) \mathrm{DAB}$ colorimetric reaction. $(\mathrm{b}, \mathrm{e})$ Computer assisted merged images; blue is DAPI nuclear staining, while red is CS-biotin contrast. Bar: a-c, $200 \mu \mathrm{m}$; d-f $50 \mu \mathrm{m}$.

determining the highest intracellular nanoparticle uptake. This result could account for a higher cytotoxicity of QDs as compared to that of CSS rods.

Immunostaining Using QR-Biotin. We tested the feasibility of using biotin-conjugated QRs as probes for tissue imaging, on rat brain tissue sections, by immunolocalization of two wellknown proteins. A neuronal specific protein, the neuronal enolase $\mathrm{NSE},{ }^{4}$ and the widely expressed cytoskeleton component actin were chosen, as their expression pattern is well reported. ${ }^{45}$ While the immunochemistry with the antibodies followed standard protocols, the fluorescence detection through QR-biotin conjugation was based on the avidin-biotin chemistry methods, similarly to the test performed on cultured cells. To this aim, brain tissue sections, following immunoreaction with primary antibodies (rabbit anti-NSE and anti-ACT) and with biotinylated secondary antibodies (antirabbit IgG) were incubated with avidin to anchor the QR-biotin (see Experimental Section for details). Results shown in Figure 8 illustrate that the protein localization

(44) Vannucchi, M. G.; Faussone-Pellegrini, M. S. J. Comp. Neurol. 2000, $425(3), 369-381$.

(45) Buno, I.; Juarranz, A.; Canete, M.; Villanueva, A.; Gosalvez, J.; Stockert, J. C. Micron 1998, 29(6), 445-449. patterns corresponded to those expected both for NSE and actin, confirming the efficiency of the CS-bioconjugate, the specificity of the interaction with avidin, and the feasibility to be used as staining agents. The extreme QR photostability and fluorescence intensity could allow imaging of the same tissue region at high resolution, over long periods of time, and in multiplexing with DAPI imaging, thus avoiding the computer assisted merging of multiple fluorescence signals (Figure 8). Although limited nonspecific binding of $\mathrm{QR}$ - biotin to tissue section was observed, alternative processing of the tissue sections with adequate blocking agents might be tried or ad hoc developed to decrease the signal-to-noise ratio, which was not in the aim of the present work.

Specific Cell Labeling with QR-Folate Conjugates. To demonstrate the selective targeting of human cells by $\mathrm{QR}$-folate (QR-FA) conjugates, human nasopharyngeal epidermal carcinoma (KB) cells, expressing the folate receptors, were incubated with the functionalized QRs. Figure 9 shows the confocal microscopy images of the cells doped for $1 \mathrm{~h}$ at $25^{\circ} \mathrm{C}$ with the $\mathrm{CS}$ and CSS conjugates at $25 \mu \mathrm{M} \mathrm{Cd}$ concentration. QR-FAs appear to accumulate in vesicles within the cells, suggesting folatemediated endocytotic uptake (Figure 9b and e, and Figure S3 


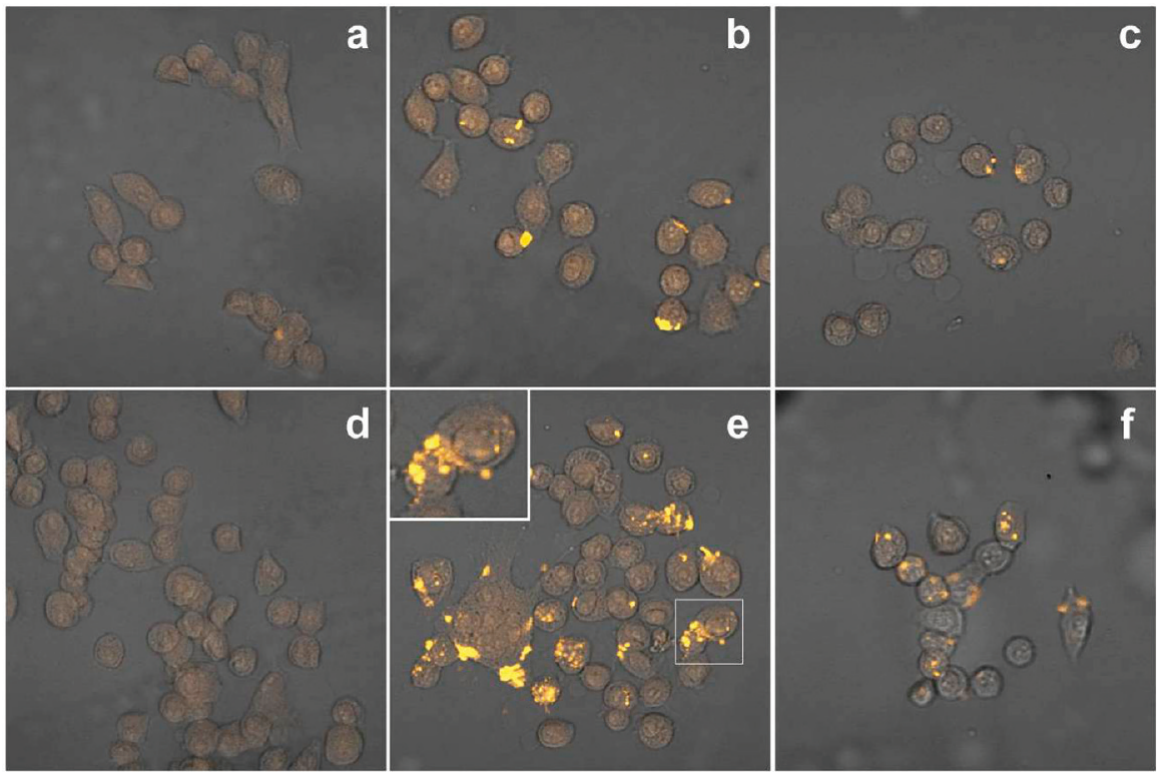

Figure 9. Confocal microscopy images of fixed KB cells incubated with CS (a-c) and CSS (d-f) samples for $1 \mathrm{~h}$ at $25 \mu \mathrm{M}$ Cd. Cells were treated with the QR-PEG conjugates ( $\mathrm{a}$ and $\mathrm{d}$ ); the QR-FA conjugates ( $\mathrm{b}$ and e); and free folic acid and subsequently the QR-FA conjugates (c and f).

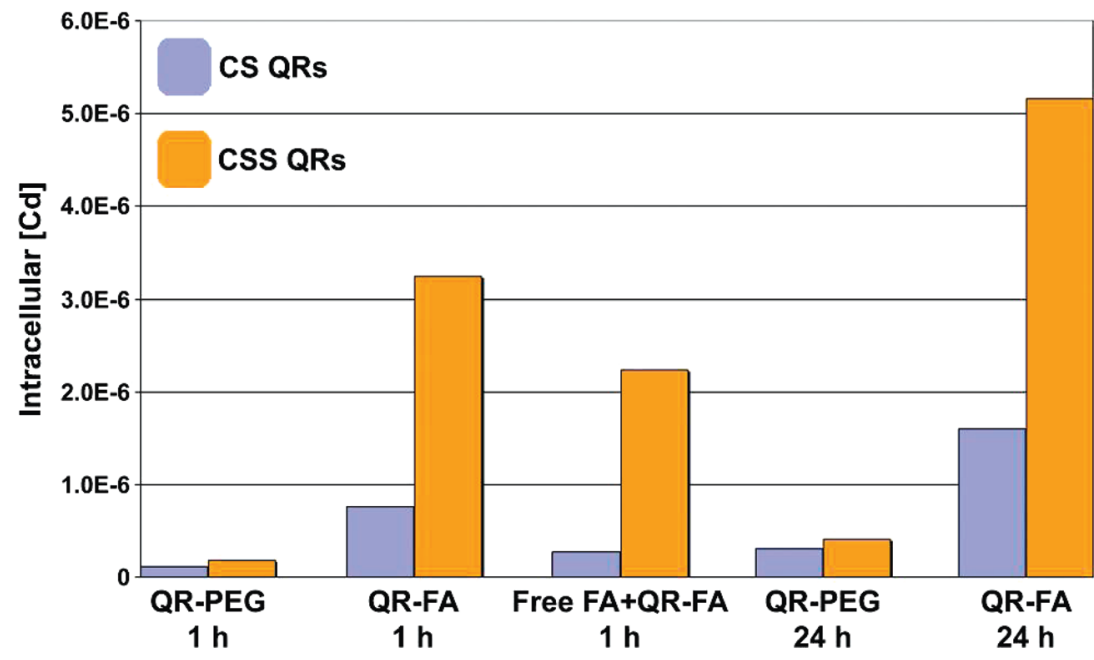

Figure 10. Comparison of the intracellular Cd concentration from cells treated with QR-FA and QR-PEG after 1 and $24 \mathrm{~h}$ of incubation time and at $25 \mu \mathrm{M}$ Cd doping amounts. A further experiment was performed, in which the competitive behavior due to the addition of free folic acid was monitored and scored as a reduction of the amount of FA-conjugate uptaken by cells.

(Supporting Information) also shows detailed bright field, confocal, and overlaid images of few cells doped with CSS-FA QRs). In a control experiment with the corresponding QR-PEG nanocrystals, only traces were taken up after $1 \mathrm{~h}$ of incubation (Figure 9a and d). Furthermore, saturating the membrane FA receptors with free folic acid before the addition of QR-FA also led to minimal nanocrystal uptake because of specific competion (Figure $9 \mathrm{c}$ and $\mathrm{f}$ ).

To quantitatively estimate the specific uptake of the folate conjugate by KB cells with respect to QR-PEG, the intracellular $\mathrm{Cd}$ concentration was determined by means of elemental analysis and normalized to the number of cells. As shown in Figure 10, $1 \mathrm{~h}$ postincubation, QR-FA bioconjugates had been taken up with higher efficiency compared to that of the diamino-PEG functionalized rods. The same trend could be observed $24 \mathrm{~h}$ postincubation, when $\mathrm{QR}-\mathrm{FA}$ reached the highest intracellular accumulation value.
As a general trend, CSS QRs were internalized more efficiently than CS QRs, which may be due to small differences in the surface coating and in the number of FA ligands bound to the NCs.

The relatively high dose of $\mathrm{Cd}$ measured in the competitive assay might depend on the rapid and continuous turnover of the folate receptors, which, following internalization of free folic acid, become again available on the membrane surface to interact with QR-FA. ${ }^{46}$

MTT assay was also performed to investigate cytotoxic effects of all QRs used in this study on the KB cells. Results of Figure S4 (Supporting Information) show similar values of cell viability (at $25 \mu \mathrm{M}$ Cd concentration), around $90 \%$, except for CS-FA QRs, presenting slightly lower value, probably because of a major Cd leakage from the core with respect to the double shielded CSS.

(46) Kamen, B. Semin. Oncol. 1997, 24(5), 30-39. 


\section{Conclusions}

In conclusion, we reported the bioconjugation of new rodshaped fluorescent nanocrystals made of $\mathrm{CdSe} / \mathrm{CdS}$ and $\mathrm{CdSe} /$ $\mathrm{CdS} / \mathrm{ZnS}$, which display enhanced photostability and higher PL quantum efficiencies compared to traditional $\mathrm{CdSe} / \mathrm{ZnS}$ QDs, thus allowing the use of lower nanoparticle concentrations for specific cell labeling experiments.

Biotin-conjugated nanorods were prepared and exploited as an alternative class of fluorescent molecular probes for cell and tissue imaging, while FA-conjugated QRs showed the potential of these materials for targeting and imaging specific tumor cells. The results presented in this study demonstrated that both types of QRs are brighter probes compared to the corresponding bioconjugate QDs, still maintaining the ability to target specific cells as efficiently as QDs. The major advantage arising from the biotin and FA-functionalized nanorods is that because of the enhanced PL emission of the samples, lower amounts of nanoparticles are required to obtain a bright fluorescence signal from the labeled cells, reducing potential toxic effects in staining applications. Furthermore, by using bioconjugated-CSS QRs we observed that the growth of an outer $\mathrm{ZnS}$ shell also contributed to the reduction of the cytotoxicity of the $\mathrm{CdSe} / \mathrm{CdS}$ rods by reducing Cd leakage, confirming what was reported by other groups on other types of fluorescent nanocrystals. ${ }^{43,47-49}$

\section{Experimental Section}

Chemicals. All chemicals were used as received. Bis(hexamethylene)triamine (\#14506), sodium tetraborate decahydrate $(\#$ S9640), boric acid (\#B1934), Tris-borate-EDTA buffer (\#T3913), sucrose (\#16104), $N$-(3-dimethylaminopropyl)- $N^{\prime}$-ethylcarbodiimide hydrochloride (EDC, \#E7750), biotin 3-sulfo$N$-hydroxysuccinimide ester (NHS-biotin, \#B5161), avidin (\#A9275), folic acid (\#F8758), 3-(4,5-dimethyl-2-thiazolyl)2,5-diphenyl-2H-tetrazolium bromide (MTT salt, \#135038), and DAPI (\#D9564) were purchased from Sigma-Aldrich. DiaminePEG 897 (\#06703) was purchased from Fluka. Streptavidin-Texas red (\#21624) was purchased from Pierce. Agarose (D-1 low EEO, \#C1349) was purchased from Eppendorf. Ultrapure grade water with a conductivity of $18.2 \mathrm{M} \Omega \mathrm{cm}$ was used in all experiments.

Synthesis of Water-Soluble Nanocrystals and Functionalization with Diamino-PEG Molecules. CdSe/ZnS QDs were prepared following standard procedures, ${ }^{50,51}$ while CdSe/CdS and $\mathrm{CdSe} / \mathrm{CdS} / \mathrm{ZnS}$ QRs were synthesized according to procedures developed by us and reported in previous papers. ${ }^{31,35}$ The $\mathrm{CdSe} / \mathrm{CdS} / \mathrm{ZnS}$ rods used in this work were directly prepared from $\mathrm{CdSe} / \mathrm{CdS}$ seeds. Both samples were exploited in this study, and this ensured that samples with similar features could be easily compared. CdSe/ZnS QDs and $\mathrm{CdSe} / \mathrm{CdS}$ and $\mathrm{CdSe} / \mathrm{CdS} / \mathrm{ZnS}$ QRs emitting in the orange/red spectrum were used in order to avoid the overlap with the autofluorescence signal of cells.

QRs were transferred from the organic phase into water by adopting a polymer coating approach. ${ }^{36}$ In order to completely remove the excess polymer from the water-soluble nanocrystals, an ultracentrifugation step was introduced, as already described by our group..$^{37}$ A Beckman Coulter Optima LE-80K ultracentrifuge, equipped with a SW41 Ti rotor, was used at 150,000 rcf and on a continuous sucrose gradient. The sucrose density range

(47) Seleverstov, O.; Zabirnyk, O.; Zscharnack, M.; Bulavina, L.; Nowicki, M.; Heinrich, J. M.; Yezhelyev, M.; Emmrich, F.; , R.; Bader, A. Nano Lett. 2006, 6(12), 2826-2832.

(48) Chan, W. H.; Shiao, N. H.; Lu, P. Z. Toxicol. Lett. 2006, 167(3), 191-200.

(49) Selvan, S. T.; Tan, T. T.; Ying, J. Y. Adv. Mater. 2005, 17(13), 1620.

(50) Dabbousi, B. O.; RodriguezViejo, J.; Mikulec, F. V.; Heine, J. R.; Mattoussi, H.; Ober, R.; Jensen, K. F.; Bawendi, M. G. J. Phys. Chem. B 1997, 101(46), 9463-9475.

(51) Peng, Z. A.; Peng, X. G. J. Am. Chem. Soc. 2001, 123(1), 183-184. and the ultracentrifugation time varied with the size and the composition of the nanoparticles: $5-30 \%$ for $4 \mathrm{~h}$ in the case of QDs and $20-66 \%$ for $2 \mathrm{~h}$ in the case of QRs which have length of $22 \mathrm{~nm}$ and a diameter of $4 \mathrm{~nm}$.

The surface carboxylic groups of the polymer were used to bind one of the amino moieties of the diamino-PEG molecules through the formation of an amide bond. ${ }^{52}$ Diamino-PEG molecules were introduced to increase the stability of the nanoparticles and to allow further surface functionalization.

Nanocrystals Conjugation to Biotin and Folic Acid. The free amino groups on the nanocrystal surface were used to covalently bind biotin or folic acid through the formation of an amide bond, by using EDC chemistry. ${ }^{52}$

Biotin-conjugated nanocrystals were prepared by adding NHSbiotin dissolved in borate buffer ( $\mathrm{pH}$ 9) to a $2 \mu \mathrm{M}$ solution of diamino-PEG nanoparticles (either QDs or both types of QRs) at a biotin/NP ratio of 500 in the case of QDs and 1,000 for QRs. Then EDC was added at the following EDC/NP ratios: 75,000 for QDs and 250,000 in the case of $\mathrm{CdSe} / \mathrm{CdS}$ and $\mathrm{CdSe} / \mathrm{CdS} / \mathrm{ZnS}$ QRs (for rods $22 \mathrm{~nm}$ long and $4 \mathrm{~nm}$ thick). After $3 \mathrm{~h}$ of vigorous stirring, excess biotin was removed by filtering the solutions through Amicon filters (10,000 and 30,000 MWCO for QDs and QRs, respectively) at least 5 times.

For the preparation of folic acid-conjugated CS and CSS QRs, folic acid $(0.01 \mu \mathrm{M})$ was dissolved in borate buffer $(1 \mathrm{~mL})$ and activated with $\mathrm{EDC}(0.02 \mu \mathrm{M})$ for $15 \mathrm{~min}$. After obtaining a clear solution, the diamino-PEG-functionalized QRs were added $(0.1 \mu \mathrm{M}$ final concentration $)$ and the mixture shaken for $6 \mathrm{~h}$ in the dark. The residue was filtered over Amicon filters $(30,000 \mathrm{MWCO})$ and then purified by ultracentrifugation at $150,000 \mathrm{rcf}$ for $2 \mathrm{~h}$ on a $20-66 \%$ sucrose density gradient.

Gel Electrophoresis Characterization. The samples were run on $2 \%$ agarose gel in TBE buffer for $1 \mathrm{~h}$ at $100 \mathrm{~V}$. Prior to gel electrophoresis, a solution containing Orange $\mathrm{G}$ and $30 \%$ glycerol in gel-loading buffer corresponding to $20 \%$ of the sample volume was added to each sample. At the end of the run, the gel was observed under UV light, and a red filter was used to detect the fluorescence of the nanocrystals.

Low-Magnification TEM. The samples were prepared by dropping a dilute solution of nanocrystals in water on carboncoated copper grids (Formvar/Carbon 300 Mesh Cu). TEM images were recorded on a JEOL Jem 1011 microscope operating at an accelerating voltage of $100 \mathrm{KV}$.

Dynamic Light Scattering (DLS) and Zeta-Potential Measurements. A Zetasizer Nano ZS90 (Malvern, USA) equipped with a $4.0 \mathrm{~mW} \mathrm{He}-\mathrm{Ne}$ laser operating at $633 \mathrm{~nm}$ and an Avalanche photodiode detector was used. Measurements were made at $25^{\circ} \mathrm{C}$ in borate saline buffered solutions $(\mathrm{pH} 9)$ of the particles.

UV-Vis Absorption and Photoluminescence Spectra. Spectra were recorded using a Varian Cary 300 UV-vis spectrophotometer and a Cary Eclipse spectrofluorimeter. Photoluminescence quantum yield measurements were performed adopting the gradient method and using Rhodamine $6 \mathrm{G}$ as reference fluorescent dye (excitation wavelength at $488 \mathrm{~nm}$ ). Briefly, solutions of Rhodamine $6 \mathrm{G}$ at different concentrations were prepared, and their optical densities at the excitation wavelength ranged from 0.01 to 0.1 to avoid self-absorption effects in the photoluminescence spectra; their absorption and PL spectra were recorded. Then the optical densities (at $488 \mathrm{~nm}$ ) and the integrated fluorescence intensities of the solutions were plotted in a graph. The series of points was then interpolated with a straight line of slope $m$ R6G and intercept equal to zero. The same approach was adopted for both nanocrystals (either QDs or QRs), yielding therefore for each type of nanocrystal an interpolation line of slope $m \mathrm{NC}$ and an intercept close to zero. The PL QY from each nanocrystal sample was then calculated using the following

(52) Williams, A.; Ibrahim, I. T. Chem. Rev. 1981, 81(4), 589-636. 
equation: $Q Y_{\mathrm{NC}}=Q Y_{\mathrm{R} 6 \mathrm{G}} m_{\mathrm{NC}} / m_{\mathrm{R} 6 \mathrm{G}}\left(\eta_{\text {solvent }} / \eta_{\text {ethanol }}\right)^{2}$ where $Q Y_{\mathrm{R} 6 \mathrm{G}}$ is the $\mathrm{QY}$ of Rhodamine $\mathrm{G} 6$ (which is known from the vendor), and $\eta_{\text {ethanol }}$ and $\eta_{\text {solvent }}$ are the refractive indices of the solvents in which the dye and the nanocrystal sample are dissolved, respectively.

Cell Culture. HeLa cells and $\mathrm{KB}$ cells were grown at $37^{\circ} \mathrm{C}$ and in a $5 \% \mathrm{CO}_{2}$ atmosphere in RPMI-1640 medium, supplemented with L-glutamine $(2 \mathrm{mM})$, penicillin (100 units $/ \mathrm{mL}$ ), streptomycin $(100 \mu \mathrm{g} / \mathrm{mL})$, and $10 \%$ heat-inactivated fetal bovine serum (FBS).

Treatment of HeLa Cells with NHS-Biotin and Avidin. HeLa cells $\left(10^{5}\right)$ were seeded in each well of a 6-well plate and, after incubation at $37^{\circ} \mathrm{C}$ for $24 \mathrm{~h}$, the medium was removed and the cells washed with PBS. NHS-biotin ( $1 \mathrm{mM}$ in PBS) was added to each well followed by incubation for $30 \mathrm{~min}$ at $4{ }^{\circ} \mathrm{C}$. The solution was then removed, and the cells were washed with PBS three times. At this point, a solution of avidin ( $10 \mu \mathrm{M}$ in PBS) was added to each well followed by incubation for $30 \mathrm{~min}$ at $4{ }^{\circ} \mathrm{C}$. Finally, the solution was removed and the cells washed with PBS three times, after which a fresh medium, containing the biotin-nanocrystal conjugates at different $\mathrm{Cd}$ concentrations (5 or $50 \mu \mathrm{M})$ was added to each well.

Confocal Microscopy Imaging of Cells. Confocal microscopy images were recorded on an Olympus FV-1000 microscope equipped with an argon laser source (excitation at $488 \mathrm{~nm}$ ) with a DM488/405-type dichroic filter and an acquisition window at $615 \pm 20 \mathrm{~nm}$. After $1 \mathrm{~h}$ of incubation at $4{ }^{\circ} \mathrm{C}$, cells were directly imaged under the confocal microscope, while after $24 \mathrm{~h}$ of incubation at $37^{\circ} \mathrm{C}$ imaging was performed on both living and fixed cells.

Determination of the Intracellular Uptake of $\mathrm{Cd}$ and Nanoparticles. To estimate the intracellular $\mathrm{Cd}$ concentration and hence the intracellular nanocrystals uptake, $10^{5}$ cells were seeded in each well ( $3,5 \mathrm{~cm}$ in diameter) of a 6 well plate. After $24 \mathrm{~h}$ of incubation at $37{ }^{\circ} \mathrm{C}$, the medium was replaced with fresh medium containing the nanocrystals at $50 \mu \mathrm{M} \mathrm{Cd}$ concentration. After 5 or $24 \mathrm{~h}$ of incubation at $37^{\circ} \mathrm{C}$, the medium was removed, the cells were washed twice with PBS ( $\mathrm{pH} 7.4)$, trypsinized, and counted using a cell-counting chamber. Then, the cell suspensions were digested using a $\mathrm{HCl} / \mathrm{HNO}_{3} 3: 1(\mathrm{v} / \mathrm{v})$ solution, and the intracellular $\mathrm{Cd}$ concentration was measured by means of elemental analysis and normalized to the number of cells. To convert the intracellular $\mathrm{Cd}$ concentration to nanoparticle concentration, the procedure described in ref 35 was employed. In detail, the average diameter and length of the nanoparticles were assessed via statistical analysis on TEM images. The average number of $\mathrm{Cd}$ atoms per nanoparticle was determined by building a structural model of the nanoparticle with the same geometrical parameters as determined by TEM. Then, knowing the average number of $\mathrm{Cd}$ atoms per nanoparticle and the total concentration of this species in solution, it is possible to determine the concentration of nanoparticles.

Cytotoxicity Assay. The MTT viability assay was performed to estimate the cytotoxicity of the conjugated nanocrystals. Specifically, $5 \times 10^{4}$ cells were first seeded in each well of a 12-well plate, and after incubation at $37^{\circ} \mathrm{C}$ for $24 \mathrm{~h}$, the medium was replaced with a fresh medium containing the nanocrystals at different $\mathrm{Cd}$ concentrations $(5$ and $50 \mu \mathrm{M})$. After additional $24 \mathrm{~h}$ of incubation at $37^{\circ} \mathrm{C}$, the medium was removed, and the cells were washed twice with PBS ( $\mathrm{pH} 7.4)$. Then $1 \mathrm{~mL}$ of a solution containing $1 \mathrm{mg} / \mathrm{mL}$ of MTT (3-(4,5-dimethylthiazol-2-yl)2,5-diphenyltetrazolium bromide) dissolved in culture medium was added to each well. After a $3 \mathrm{~h}$ incubation at $37^{\circ} \mathrm{C}$, the MTT, reduced by the mitochondrial reductase of vital cells, formed a dark insoluble product, formazan. At this point, the medium was collected from each well, centrifuged, and the supernatant discarded. The dark pellet was dissolved in DMSO, leading to a violet solution, the absorbance of which at $570 \mathrm{~nm}$ was determined. The absorbance value can be correlated to the percentage of vital cells, by comparing the data of the doped cells with those of control cells (i.e., with no conjugates added to medium).

Immunohysto Chemistry. Rat brain slices (14 $\mu \mathrm{m}$ thick) were a generous gift of L. Cristino. Adult male Wistar rats were deeply anesthetized and perfused transcardially with $3.5 \%$ paraformaldehyde and $1 \%$ picric acid in $0.1 \mathrm{M}$ phosphate buffer (PB). Tissue blocks containing brain and cerebellum were cryoprotected in $20 \%$ sucrose in $0.1 \mathrm{M} \mathrm{PB}$ overnight at $4{ }^{\circ} \mathrm{C}$, transferred in frozen section medium (OCT), frozen at $-80{ }^{\circ} \mathrm{C}$, and cut on a freezing microtome into $14-\mu \mathrm{m}$-thick sections. The sections were transferred on superfrost slides. For immunostaining, tissue sections were blocked in $1 \%$ Normal Goat Serum and $0.4 \%$ triton in TBS $(0.1 \mathrm{M}$ Tris base $+0.15 \mathrm{M} \mathrm{NaCl}$ at $\mathrm{pH} 7.3)$, and incubated with specific antibodies for $16 \mathrm{~h}$ at $4{ }^{\circ} \mathrm{C}$, diluted as follows: rabbit antiNSE (Chemicon), 1:1000 [provided by Dr. A. Sadile, University of Naples]; rabbit anti-Actin (Sigma), 1:100. After 3 washes with TBS, sections were incubated with biotinylated goat antirabbit (1:100). After washes, the sections were incubated with $20 \mu \mathrm{gr} / \mathrm{mL}$ Avidin (Sigma A9390) in TBS for $1 \mathrm{~h}$. Finally, $20 \mathrm{nM} \mathrm{CS}-$ biotin was used for fluorescence detection. Fluorescently labeled tissues were counterstained with $4^{\prime}, 6$-diamidino-2-phenylindole dihydrochloride (DAPI; $0.25 \mu \mathrm{g} / \mathrm{mL}$ ) and mounted with Aquatex medium (Merk). Fluorescence microscopy was accomplished by using an inverted microscope (Axiovert 100, Zeiss) equipped with a digital color camera (Olympus, DP70) and fluorescence filter sets (BP450-490/FT510/LP515). Bright field immunohistochemistry were developed using the $\mathrm{ABC}$ kit (Vector), and finally incubated with $0.05 \%$ diaminobenzidine and $0.0006 \%$ hydrogen peroxide. For imaging acquisition and analysis, the software system Cell F (Olympus) was used.

Supporting Information Available: DLS and zeta-potential characterization of QDs, CS-QRs, and CSS-QRs for biotin conjugation and UV-vis and PL spectra of water-soluble and folic acid functionalized QDs, CS-QRs, and CSS-QRs are reported. Additional confocal images of cells at higher magnification and MTT cytotoxicity results are also reported. This material is available free of charge via the Internet at http:// pubs.acs.org. 